Research Article

\title{
Gastrointestinal Pathobionts in Pediatric Crohn's Disease Patients
}

\author{
Rachel V. Purcell $\mathbb{D}^{1},{ }^{1}$ Nadeem O. Kaakoush $\mathbb{D}^{2},{ }^{2}$ Hazel M. Mitchell $\mathbb{D},{ }^{3}$ John F. Pearson, ${ }^{4}$ \\ and Jacqueline I. Keenan ${ }^{1}$ \\ ${ }^{1}$ Department of Surgery, University of Otago, Christchurch, New Zealand \\ ${ }^{2}$ School of Medical Sciences, University of New South Wales, Sydney, NSW, Australia \\ ${ }^{3}$ School of Biotechnology and Biomolecular Sciences, University of New South Wales, Sydney, NSW, Australia \\ ${ }^{4}$ Biostatistics and Computational Biology Unit, University of Otago, Christchurch, New Zealand
}

Correspondence should be addressed to Rachel V. Purcell; rachel.purcell@otago.ac.nz

Received 18 March 2018; Accepted 13 June 2018; Published 17 July 2018

Academic Editor: Joseph Falkinham

Copyright (C) 2018 Rachel V. Purcell et al. This is an open access article distributed under the Creative Commons Attribution License, which permits unrestricted use, distribution, and reproduction in any medium, provided the original work is properly cited.

\begin{abstract}
Crohn's disease (CD) is an inflammatory disease of the gastrointestinal tract, with a rising incidence worldwide, particularly in children. CD is thought to arise due to an immune response to environmental factors. The role of bacteria in CD has recently been highlighted, and here, we examine the prevalence of two bacterial species, enterotoxigenic Bacteroides fragilis (ETBF) and Fusobacterium nucleatum, implicated in gastrointestinal pathologies, in a pediatric CD cohort. Stool samples from 30 children with treatment-naïve CD and 30 age- and sex-matched controls were collected, and DNA was extracted. Quantitative PCR was used to determine the levels of ETBF and F. nucleatum in stool samples. Bacterial positivity and relative abundance were assessed between cases and controls and in relation to disease severity. No associations were found between colonization with ETBF and $\mathrm{CD}$, or between colonization with either ETBF or F. nucleatum and disease severity or presence of C. concisus. However, a strong association was observed between positivity for F. nucleatum in the stool samples and the occurrence of CD in patients (25/30) as compared to controls $(8 / 30)(P=0.003)$. F. nucleatum is more prevalent in the stool samples of pediatric CD patients, compared to healthy controls, and may have potential use as a biomarker of pediatric CD.
\end{abstract}

\section{Background}

Inflammatory bowel diseases (IBDs) are characterized by chronic inflammation in the gastrointestinal tract (GIT), and the two most commonly described forms of IBDs are Crohn's disease (CD) and ulcerative colitis (UC). CD is characterized by inflammatory lesions, which may be transmural, in any location in the GIT, with granulomas being a common feature. Although the etiology of $\mathrm{CD}$ is, as yet, unknown, there is evidence that dysregulated immune response to environmental triggers may lead to $\mathrm{CD}$ onset in genetically predisposed individuals.

CD affects around 3.2/1000 people in Europe and North America, and the incidence is rising, particularly in children. While previously the incidence of CD in children resident in nonindustrialized countries has been low, current evidence would suggest that, with industrialization, the incidence of $\mathrm{CD}$ is increasing [1].

$\mathrm{CD}$ is associated with a slightly lower life expectancy and considerable morbidity. Patients with long-standing $\mathrm{CD}$ located in the colon have a similar increased risk of colorectal cancer development as do those with UC $[2,3]$, and as a result, there has been intense interest in identifying potential etiological agents of the disease. The contribution of microbial dysbiosis to inflammation in the gut has recently been described, and several studies have identified changes in bacterial communities and particular species that are associated with CD. Decreased abundance of beneficial bacterial species from the Lachnospiraceae and Ruminococcaceae families and increased abundance of potential 
TABle 1: Primers and probes used for quantitative PCR.

\begin{tabular}{|c|c|c|}
\hline Gene & Sequence $5^{\prime} \rightarrow 3^{\prime}$ & Reference \\
\hline F. nucleatum nusG & & [19] \\
\hline Forward primer & CAACCATTACTTTAACTCTACCATGTTCA & \\
\hline Reverse primer & GTTGACTTTACAGAAGGAGATTATGTAAAAATC & \\
\hline Probe & TCAGCAACTTGTCCTTCTTGATCTTTAAATGAACC & \\
\hline$B f t$ & & {$[20]$} \\
\hline Forward primer & GGATAAGCGTACTAAAATACAGCTGGAT & \\
\hline Reverse primer & CTGCGAACTCATCTCCCAGTATAAA & \\
\hline Probe & CAGACGGACATTCTC & \\
\hline
\end{tabular}

pathobionts, such as Campylobacter spp. and Fusobacterium nucleatum, have been identified using metagenomic and targeted studies of CD patients [4-7]. Both C. concisus and F. nucleatum are oral pathogens with epithelial cell adherence and invasive potential $[4,8-10]$; certain strains of $C$. concisus produce exotoxin 9 and/or zonula occludens toxin (ZOT) [11]. Enterotoxigenic Bacteroides fragilis (ETBF) produces a zinc metalloprotease toxin called the Bacteroides fragilis toxin (Bft) that has been shown to induce colonic inflammation in mice $[12,13]$ and is associated with diarrheal disease and the development of colorectal cancer [14-16].

In this study, we investigate the abundance of ETBF and F. nucleatum in a cohort of pediatric Crohn's disease patients and age-matched controls. We also examined the association of these bacteria with the presence of previously determined C. concisus and its toxins, as well as with patient characteristics.

\section{Materials and Methods}

2.1. Study Subjects. Thirty children with Crohn's disease, diagnosed at Sydney Children's Hospital (Randwick, Australia), were recruited, in addition to 30 healthy controls. Stool samples were collected from the patients prior to treatment, and exclusion criteria included antibiotic or antiinflammatory treatment in the four weeks prior to sample collection. This patient cohort has previously been described [6], and the levels of C. concisus and ZOT have previously been reported [17] in this cohort. Informed consent was obtained from all participants (or their parent/guardian), and the study was approved by the Research Ethics Committee of the University of New South Wales and the South East Sydney Area Health Service-Eastern Section, Sydney (Ethics nos. $03 / 163,03 / 165$, and 06/164).

2.2. Reference Strains. ETBF strain VPI 13784 [18] was generously supplied by Professor Cynthia Sears, Baltimore, USA, and was used as a reference strain in this study. This was cultured anaerobically on sheep blood agar (Fort Richard Laboratories). F. nucleatum was sourced from the American Type Culture Collection (ATCC) strain 25586. DNA was extracted from colonies recovered from the plates using the DNeasy Blood \& Tissue Mini Kit (Qiagen, Hilden, Germany), as per the manufacturer's instructions. DNA extraction included digestion with Proteinase $\mathrm{K}$ for 3 hours at $56^{\circ} \mathrm{C}$. The samples were stored at $-20^{\circ} \mathrm{C}$.
2.3. Primer Design. TaqMan primer/probe sets were designed to amplify regions of the $B f t$ gene of ETBF and the nus $G$ gene of $F$. nucleatum. Primer and probe sequences are shown in Table 1.

2.4. Quantitation of Genomic DNA in Bacterial Reference Strain Samples. Digital PCR (dPCR) was used to determine the concentration of bacterial genomes in each reference sample. The QuantStudio 3D Digital PCR System (Life Technologies) was used to carry out dPCR. Samples, consisting of $9 \mu \mathrm{l}$ of QuantStudio 3D Master Mix, $1.8 \mu \mathrm{l}$ of genomic DNA (25-35 ng/ $\mu \mathrm{l}$ of DNA), and $0.9 \mu \mathrm{l}$ of TaqMan primer/probe set (final concentrations of $900 \mathrm{nM} / 250 \mathrm{nM}$, resp.), in a final volume of $18 \mu \mathrm{l}$, were loaded onto chips, and thermal cycling was carried out on a GeneAmp( PCR System 9700 thermocycler (Applied Biosystems), as previously described [21]. The chips were then loaded onto the QuantStudio $^{\mathrm{TM}}$ 3D Digital PCR Instrument, and the endpoint fluorescence of each partition on the chips was measured using QuantStudio 3D Digital PCR software v3.0. Each sample was carried out in duplicate, and a no-template control was included with each run. Serial dilutions of each reference DNA sample were made to construct standard curves to determine levels of bacteria in test samples.

2.5. Quantitative PCR. DNA was extracted from fecal samples from CD cases and controls using the QIAamp DNA Stool Mini Kit (Qiagen, Hilden, Germany), as per the manufacturer's instructions. Levels of each bacterium were measured from genomic DNA samples using TaqMan probes on a LightCycler ${ }^{\circledR} 480$ thermocycler (Roche Diagnostics, Indianapolis, IN, USA), as detailed in the Methods section of the study of Purcell et al. [22]. In brief, each reaction consisted of 25-35 ng of genomic DNA, $5 \mu$ l of TaqMan Fast Advanced Master Mix (Applied Biosystems), and $0.5 \mu \mathrm{l}$ of TaqMan primer/probe (Thermo Fisher Scientific) in a $10 \mu \mathrm{l}$ reaction. Thermal cycling conditions were as follows: 1 cycle at $95^{\circ} \mathrm{C}$ for $10 \mathrm{~min}$, followed by 50 cycles at $95^{\circ} \mathrm{C}$ for $10 \mathrm{sec}$ and $60^{\circ} \mathrm{C}$ for $30 \mathrm{sec}$. All reactions were performed in triplicate.

2.6. Statistical Analysis. Fisher's exact tests, with $95 \%$ confidence intervals, were used to test for associations between Crohn's disease and the presence or absence of bacterial species. Pediatric CD activity index (PCDAI) values in the $\mathrm{CD}$ cohort were compared between patients tested negative 
and positive for each bacterial transcript, using $t$-tests, with Satterthwaite's adjustment for unequal variances and linear models, including covariates for age and gender. $P$ values for association of $\mathrm{CD}$ and PCDAI values were adjusted for multiple testing using the Benjamini and Yekutieli method [23]. All analyses were carried out using $\mathrm{R}$ version 3.2.1 (Vienna, Austria).

\section{Results}

This study used a cohort of 30 newly diagnosed pediatric CD patients, of whom 20 were male; the patients ranged in age from 3 to 17.1 years (median $=11.4$ years). Thirty healthy controls were also recruited, half of whom were male. The disease severity in patients was measured using the pediatric Crohn's disease activity index (PCDAI) and ranged from 7.5-70. Thirteen patients had mild disease $(\mathrm{PCDAI}<30$ ), while the remaining 17 patients had moderate-to-severe $\mathrm{CD}$ $(\mathrm{PCDAI} \geq 30)$. All but one patient had evidence of colonic involvement, and as a result, we were unable to analyze bacterial abundance by disease location. Previous analysis of C. concisus and its toxins, exotoxin 9 and ZOT, showed that while the numbers of children tested positive for $C$. concisus were similar between cases and controls, the relative abundance of the bacteria and exotoxin 9 was higher in $\mathrm{CD}$ patients [6].

3.1. Association of Disease Severity with Presence or Absence of $q P C R$ Products. Firstly, the distribution of PCDAI values was analyzed among the disease cohort using the Shapiro-Wilk test; there was no evidence that PCDAI was not normally distributed among $\mathrm{CD}$ patients in the cohort $(P=0.646$; Figure 1). No evidence of an association between $\mathrm{CD}$ severity, as measured by the PCDAI, and the presence or relative abundance of any bacterial transcripts or toxins was observed (all adjusted $P$ values $>0.1$; Table 2). This is similar to findings reported with C. concisus.

\subsection{Association of $C D$ and Bacterial Transcripts in Fecal} Samples. Quantitative PCR revealed that two patients and three controls were positive for ETBF, while 25 patients with $\mathrm{CD}$ were positive for $F$. nucleatum, compared with only eight controls. Analysis of associations between bacterial transcript positivity and the presence of $C D$, using Fisher's exact test, found a strong association between $F$. nucleatum positivity in stool samples and the occurrence of $\mathrm{CD}$. The $P$ value was 0.00002 , and when adjusted for age and gender, it was 0.0003 . There was no significant difference or trend in the relative abundance of $F$. nucleatum between cases and controls.

\section{Discussion}

In our study, we have investigated the prevalence and abundance of specific bacterial species in a cohort of pediatric Crohn's disease patients and matched controls. The study was conducted on fecal stool samples from newly diagnosed patients prior to treatment, and information on disease

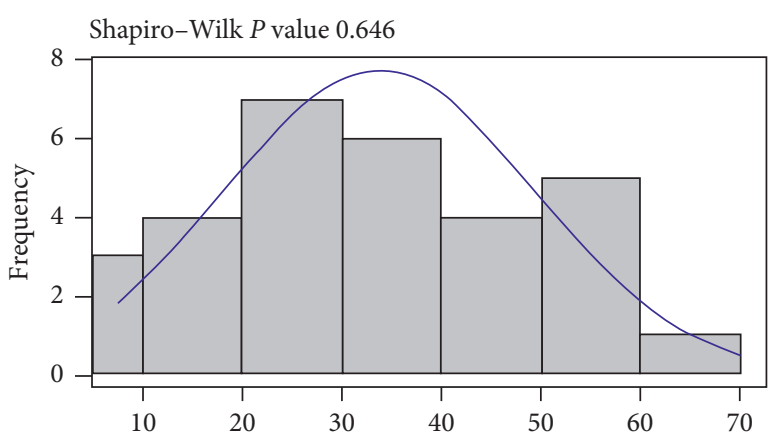

FIGURE 1: Histogram showing no evidence that disease severity, as measured by the pediatric Crohn's disease activity index (PCDAI), is not normally distributed.

severity and the abundance of $C$. concisus and its toxins was known prior to the study being carried out. Microbial dysbiosis, involving a reduction in fecal bacterial diversity, has been widely reported in gastrointestinal diseases, including $\mathrm{CD}$, ulcerative colitis, and colorectal cancer (CRC) [24-27], and there has been much focus on identifying particular bacterial species that may play a role in disease pathogenesis, as therapeutic targets and biomarkers of disease.

Bacteroides fragilis is a common gut commensal in adults, although the rate of carriage is not well characterized in children. Enterotoxigenic strains of $B$. fragilis (ETBF) secrete a proinflammatory metalloprotease toxin and have been associated with diarrheal disease in both children and adults. ETBF has also been proposed as a potential initiator of CRC via E-cadherin cleavage and NF-kB activation, as demonstrated in in vitro and animal studies and increased carriage of the bacterium in stool samples of CRC patients compared to controls $[16,28]$. In contrast, the evidence linking ETBF to IBD is limited, although a recent publication has demonstrated increased carriage of ETBF in patients with UC, compared to controls [29]. In this study, we found no association between the presence or abundance of toxigenic strains of $B$. fragilis in the stool samples and the occurrence of $\mathrm{CD}$ in patients as compared to healthy controls.

Intestinal colonization with oral bacteria has recently been implicated in inflammatory bowel diseases [5], with toxin-producing Campylobacter species being associated with pediatric $\mathrm{CD}$ as compared to healthy controls in this cohort and others $[6,17,30]$.

Fusobacterium nucleatum is another prevalent oral bacterium found in extraoral diseases and has the ability to adhere to and invade epithelial cells via its surface adhesin molecule, FadA. Strains of F. nucleatum isolated from IBD have been shown to be significantly more invasive than strains from healthy tissue or controls [4], and colonization of epithelial cells by F. nucleatum is associated with an inflammatory response [31-33]. Although causality has yet to be established, increased abundance of this bacterium has frequently been associated with CRC $[19,34,35]$ and IBD, including $\mathrm{CD}$, a known risk factor for CRC. Two separate microbiome studies of pediatric $\mathrm{CD}(n=19$ patients in both studies) found enrichment of Fusobacterium sp. in fecal samples of patients compared to healthy controls. The study 
TABLE 2: Association of PCDAI and transcripts in the gut.

\begin{tabular}{lcccccc}
\hline & Negative mean & Positive mean & Negative SE & Positive SE & $P$ value & $P$ value $($ adj.) \\
\hline Cc & 27.500 & 35.292 & 3.416 & 3.375 & 0.124 & 0.345 \\
ZOT & 29.375 & 35.318 & 4.324 & 3.505 & 0.301 & 0.749 \\
ETBF & 33.685 & 34.167 & 3.126 & 3.333 & 0.919 & 0.319 \\
Fn & 35.500 & 33.380 & 7.045 & 3.140 & 0.793 & 0.768 \\
\hline
\end{tabular}

Cc, Campylobacter concisus; ZOT, zonula occludens toxin; ETBF, enterotoxigenic Bacteroides fragilis; Fn, Fusobacterium nucleatum; SE, standard error; adj., adjusted for age and gender.

by Shaw et al. found that Fusobacterium sp. were more abundant in cases compared to controls and between nonresponders and responders to immunomodulatory treatment [36], while the study by Kaakoush et al. [17] found Fusobacterium sp. in 8/19 CD patients compared to $1 / 19$ controls. Although these studies used a $16 \mathrm{~S}$ rRNA sequencing approach that often does not allow for discrimination to the species level, the findings are similar to those of our direct bacterial species testing using qPCR in this study, where we found a strong association of $F$. nucleatum with $\mathrm{CD}$ as compared to control samples. F. nucleatum was detected in $83.3 \%$ of CD cases compared to $26.7 \%$ of controls, with similar abundance of the bacterium in samples from the two groups.

Testing for F. nucleatum in fecal samples, in combination with fecal immunochemical testing (FIT), improves detection of adenomas and CRC compared to FIT alone $[37,38]$, and our findings indicate that this oral pathogen may also prove to be a useful tool in noninvasive fecal testing for CD. A study by Dharmani et al. has reported that different strains of F. nucleatum vary in relation to their inflammatory and invasive potential [10]. This strain variance should be taken into account in future clinical studies.

Future directions would include validation of different strains of $F$. nucleatum as markers of disease in a larger independent cohort and testing the utility of F. nucleatum as a marker of disease activity. F. nucleatum has recently been linked to chemoresistance in colorectal cancer [39], and its influence on response to therapy may also have relevance in the treatment of CD. The strong association of the bacteria with CD patients compared to controls also provides direction for future functional studies on the pathogenesis and progression of $\mathrm{CD}$ using in vitro and in vivo models.

\section{Conclusions}

F. nucleatum is more prevalent in the stool samples of pediatric Crohn's disease patients, compared to healthy controls, and may have potential use as a biomarker of pediatric CD.

\section{Data Availability}

The qPCR data used to support the findings of this study are available from the corresponding author upon request.

\section{Conflicts of Interest}

The authors declare no conflicts of interest.

\section{References}

[1] S. C. Ng, H. Y. Shi, N. Hamidi et al., "Worldwide incidence and prevalence of inflammatory bowel disease in the 21st century: a systematic review of population-based studies," The Lancet, vol. 390, no. 10114, pp. 2769-2778, 2017.

[2] D. B. Sachar, "Cancer in Crohn's disease: dispelling the myths," Gut, vol. 35, no. 11, pp. 1507-1508, 1994.

[3] A. C. von Roon, G. Reese, J. Teare, V. Constantinides, A. W. Darzi, and P. P. Tekkis, "The risk of cancer in patients with Crohn's disease," Diseases of the Colon and Rectum, vol. 50, no. 6, pp. 839-855, 2007.

[4] J. Strauss, G. G. Kaplan, P. L. Beck et al., "Invasive potential of gut mucosa-derived Fusobacterium nucleatum positively correlates with IBD status of the host," Inflammatory Bowel Diseases, vol. 17, no. 9, pp. 1971-1978, 2011.

[5] D. Gevers, S. Kugathasan, L. A. Denson et al., "The treatmentnaive microbiome in new-onset Crohn's disease," Cell Host and Microbe, vol. 15, no. 3, pp. 382-392, 2014.

[6] N. O. Kaakoush, N. Castano-Rodriguez, A. S. Day, D. A. Lemberg, S. T. Leach, and H. M. Mitchell, "Campylobacter concisus and exotoxin 9 levels in paediatric patients with Crohn's disease and their association with the intestinal microbiota," Journal of Medical Microbiology, vol. 63, no. 1, pp. 99-105, 2014.

[7] N. O. Kaakoush, A. S. Day, K. D. Huinao et al., "Microbial dysbiosis in pediatric patients with Crohn's disease," Journal of Clinical Microbiology, vol. 50, no. 10, pp. 3258-3266, 2012.

[8] S. M. Man, N. O. Kaakoush, S. T. Leach et al., "Host attachment, invasion, and stimulation of proinflammatory cytokines by Campylobacter concisus and other non-Campylobacter jejuni Campylobacter species," Journal of Infectious Diseases, vol. 202, no. 12, pp. 1855-1865, 2010.

[9] S. M. Man, N. O. Kaakoush, and H. M. Mitchell, "The role of bacteria and pattern-recognition receptors in Crohn's disease," Nature Reviews Gastroenterology and Hepatology, vol. 8, no. 3, pp. 152-168, 2011.

[10] P. Dharmani, J. Strauss, C. Ambrose, E. Allen-Vercoe, and K. Chadee, "Fusobacterium nucleatum infection of colonic cells stimulates MUC2 mucin and tumor necrosis factor alpha," Infection and Immunity, vol. 79, no. 7, pp. 2597-2607, 2011.

[11] N. O. Kaakoush, S. M. Man, S. Lamb et al., "The secretome of Campylobacter concisus," FEBS Journal, vol. 277, no. 7, pp. 1606-1617, 2010.

[12] S. Rabizadeh, K. J. Rhee, S. Wu et al., "Enterotoxigenic Bacteroides fragilis: a potential instigator of colitis," Inflammatory Bowel Diseases, vol. 13, no. 12, pp. 1475-1483, 2007.

[13] K. J. Rhee, S. Wu, X. Wu et al., "Induction of persistent colitis by a human commensal, enterotoxigenic Bacteroides fragilis, in wild-type C57BL/6 mice," Infection and Immunity, vol. 77, no. 4, pp. $1708-1718,2009$.

[14] C. L. Sears, "The toxins of Bacteroides fragilis," Toxicon, vol. 39, no. 11, pp. 1737-1746, 2001. 
[15] A. Boleij, E. M. Hechenbleikner, A. C. Goodwin et al., "The Bacteroides fragilis toxin gene is prevalent in the colon mucosa of colorectal cancer patients," Clinical Infectious Diseases, vol. 60 , no. 2, pp. 208-215, 2015.

[16] J. I. Keenan, A. Aitchison, R. V. Purcell, R. Greenlees, J. F. Pearson, and F. A. Frizelle, "Screening for enterotoxigenic Bacteroides fragilis in stool samples," Anaerobe, vol. 40, pp. 50-53, 2016.

[17] N. O. Kaakoush, N. Castano-Rodriguez, A. S. Day, D. A. Lemberg, S. T. Leach, and H. M. Mitchell, "Faecal levels of zonula occludens toxin in paediatric patients with Crohn's disease and their association with the intestinal microbiota," Journal of Medical Microbiology, vol. 64, no. 3, pp. 303-306, 2015.

[18] J. S. Moncrief, A. J. Duncan, R. L. Wright, L. A. Barroso, and T. D. Wilkins, "Molecular characterization of the fragilysin pathogenicity islet of enterotoxigenic Bacteroides fragilis," Infection and Immunity, vol. 66, pp. 1735-1739, 1998.

[19] L. Flanagan, J. Schmid, M. Ebert et al., "Fusobacterium nucleatum associates with stages of colorectal neoplasia development, colorectal cancer and disease outcome," European Journal of Clinical Microbiology and Infectious Diseases, vol. 33, no. 8, pp. 1381-1390, 2014.

[20] L. A. Chen, S. Van Meerbeke, E. Albesiano et al., "Fecal detection of enterotoxigenic Bacteroides fragilis," European Journal of Clinical Microbiology and Infectious Diseases, vol. 34, no. 9, pp. 1871-1877, 2015.

[21] R. V. Purcell, J. Pearson, F. A. Frizelle, and J. I. Keenan, "Comparison of standard, quantitative and digital PCR in the detection of enterotoxigenic Bacteroides fragilis," Scientific Reports, vol. 6, no. 1, p. 34554, 2016.

[22] R. V. Purcell, J. Pearson, A. Aitchison, L. Dixon, F. A. Frizelle, and J. I. Keenan, "Colonization with enterotoxigenic Bacteroides fragilis is associated with early-stage colorectal neoplasia," Plos One, vol. 12, no. 2, Article ID e0171602, 2017.

[23] Y. Benjamini, D. Drai, G. Elmer, N. Kafkafi, and I. Golani, "Controlling the false discovery rate in behavior genetics research," Behavioural Brain Research, vol. 125, no. 1-2, pp. 279-284, 2001.

[24] R. Hansen, R. K. Russell, C. Reiff et al., "Microbiota of de-novo pediatric IBD: increased Faecalibacterium prausnitzii and reduced bacterial diversity in Crohn's but not in ulcerative colitis," American Journal of Gastroenterology, vol. 107, no. 12, pp. 1913-1922, 2012.

[25] A. W. Walker, J. D. Sanderson, C. Churcher et al., "Highthroughput clone library analysis of the mucosa-associated microbiota reveals dysbiosis and differences between inflamed and non-inflamed regions of the intestine in inflammatory bowel disease," BMC Microbiology, vol. 11, no. 1, p. 7, 2011 .

[26] J. Ahn, R. Sinha, Z. Pei et al., "Human gut microbiome and risk for colorectal cancer," Journal of the National Cancer Institute, vol. 105, no. 24, pp. 1907-1911, 2013.

[27] W. Huipeng, G. Lifeng, G. Chuang, Z. Jiaying, and C. Yuankun, "The differences in colonic mucosal microbiota between normal individual and colon cancer patients by polymerase chain reaction-denaturing gradient gel electrophoresis," Journal of Clinical Gastroenterology, vol. 48, no. 2, pp. 138-144, 2014.

[28] N. U. Toprak, A. Yagci, B. M. Gulluoglu et al., "A possible role of Bacteroides fragilis enterotoxin in the aetiology of colorectal cancer," Clinical Microbiology and Infection, vol. 12, no. 8, pp. 782-786, 2006.
[29] S. Zamani, S. Hesam Shariati, M. R. Zali et al., "“'Detection of enterotoxigenic Bacteroides fragilis in patients with ulcerative colitis," Gut Pathogens, vol. 9, no. 1, p. 53, 2017.

[30] S. Kugathasan, L. A. Denson, T. D. Walters et al., "Prediction of complicated disease course for children newly diagnosed with Crohn's disease: a multicentre inception cohort study," The Lancet, vol. 389, no. 10080, pp. 1710-1718, 2017.

[31] A. N. McCoy, F. Araujo-Perez, A. Azcarate-Peril, J. J. Yeh, R. S. Sandler, and T. O. Keku, "Fusobacterium is associated with colorectal adenomas," PLoS One, vol. 8, no. 1, Article ID e53653, 2013.

[32] Y. W. Han, W. Shi, G. T. Huang et al., "Interactions between periodontal bacteria and human oral epithelial cells: Fusobacterium nucleatum adheres to and invades epithelial cells," Infection and Immunity, vol. 68, no. 6, pp. 3140-3146, 2000.

[33] S. R. Park, D. J. Kim, S. H. Han et al., "Diverse toll-like receptors mediate cytokine production by Fusobacterium nucleatum and Aggregatibacter actinomycetemcomitans in macrophages," Infection and Immunity, vol. 82, no. 5, pp. 1914-1920, 2014.

[34] M. Castellarin, R. L. Warren, J. D. Freeman et al., "Fusobacterium nucleatum infection is prevalent in human colorectal carcinoma," Genome Research, vol. 22, no. 2, pp. 299-306, 2012.

[35] A. D. Kostic, D. Gevers, C. S. Pedamallu et al., "Genomic analysis identifies association of Fusobacterium with colorectal carcinoma," Genome Research, vol. 22, no. 2, pp. 292-298, 2012.

[36] K. A. Shaw, M. Bertha, T. Hofmekler et al., "Dysbiosis, inflammation, and response to treatment: a longitudinal study of pediatric subjects with newly diagnosed inflammatory bowel disease,” Genome Medicine, vol. 8, no. 1, p. 75, 2016.

[37] S. H. Wong, T. N. Kwong, T. C. Chow et al., "Quantitation of faecal Fusobacterium improves faecal immunochemical test in detecting advanced colorectal neoplasia," Gut, vol. 66, no. 8, pp. 1441-1448, 2016.

[38] N. T. Baxter, J. P. Zackular, G. Y. Chen, and P. D. Schloss, "Structure of the gut microbiome following colonization with human feces determines colonic tumor burden," Microbiome, vol. 2, no. 1, p. 20, 2014.

[39] T. Yu, F. Guo, Y. Yu et al., "Fusobacterium nucleatum promotes chemoresistance to colorectal cancer by modulating autophagy," Cell, vol. 170, pp. 548-563, 2017. 


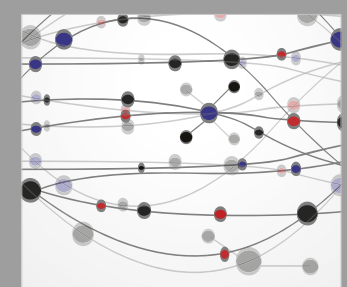

The Scientific World Journal
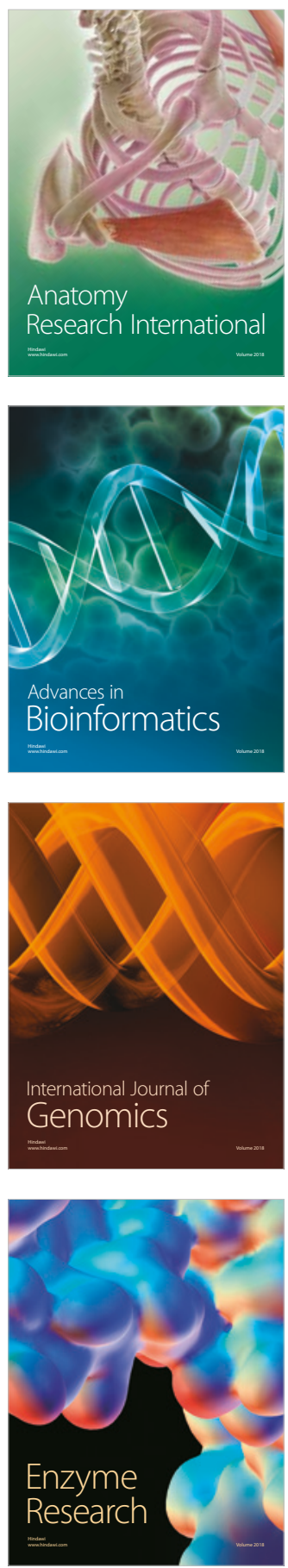
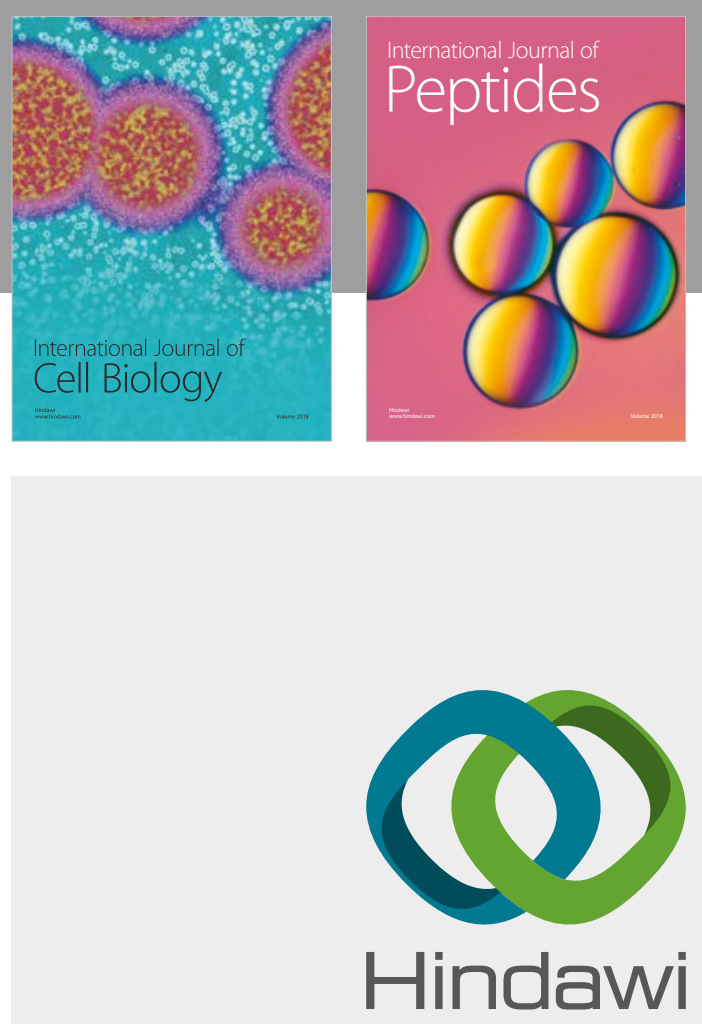

Submit your manuscripts at

www.hindawi.com
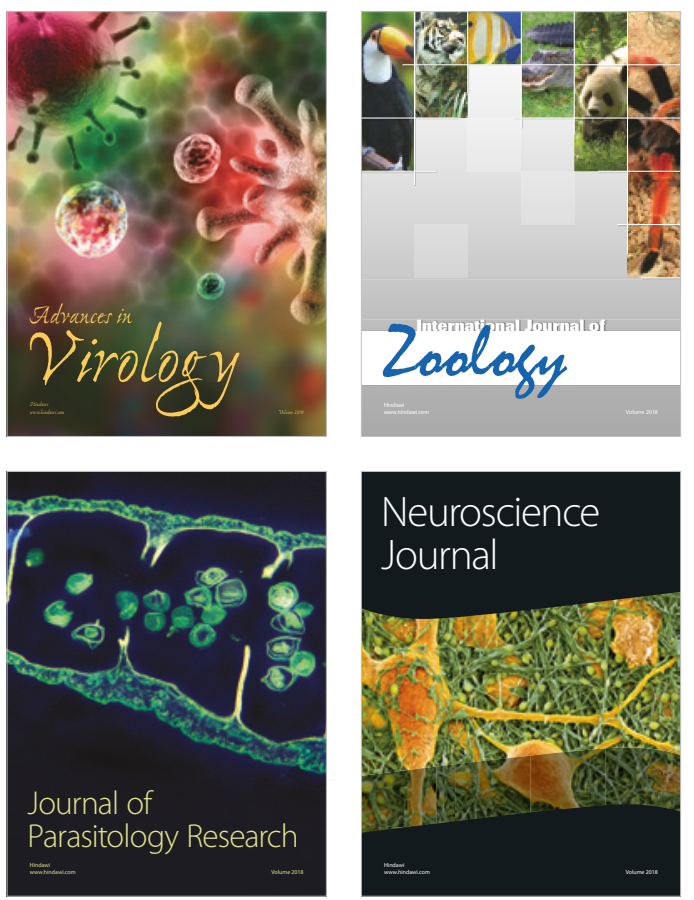
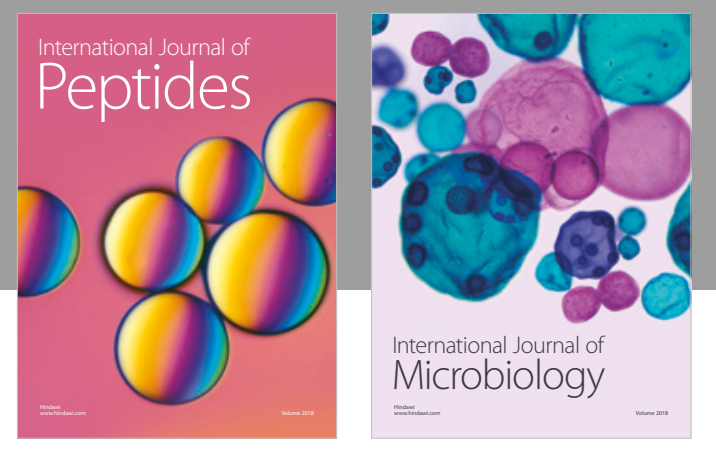

nternational Journal of Microbiology
Journal of
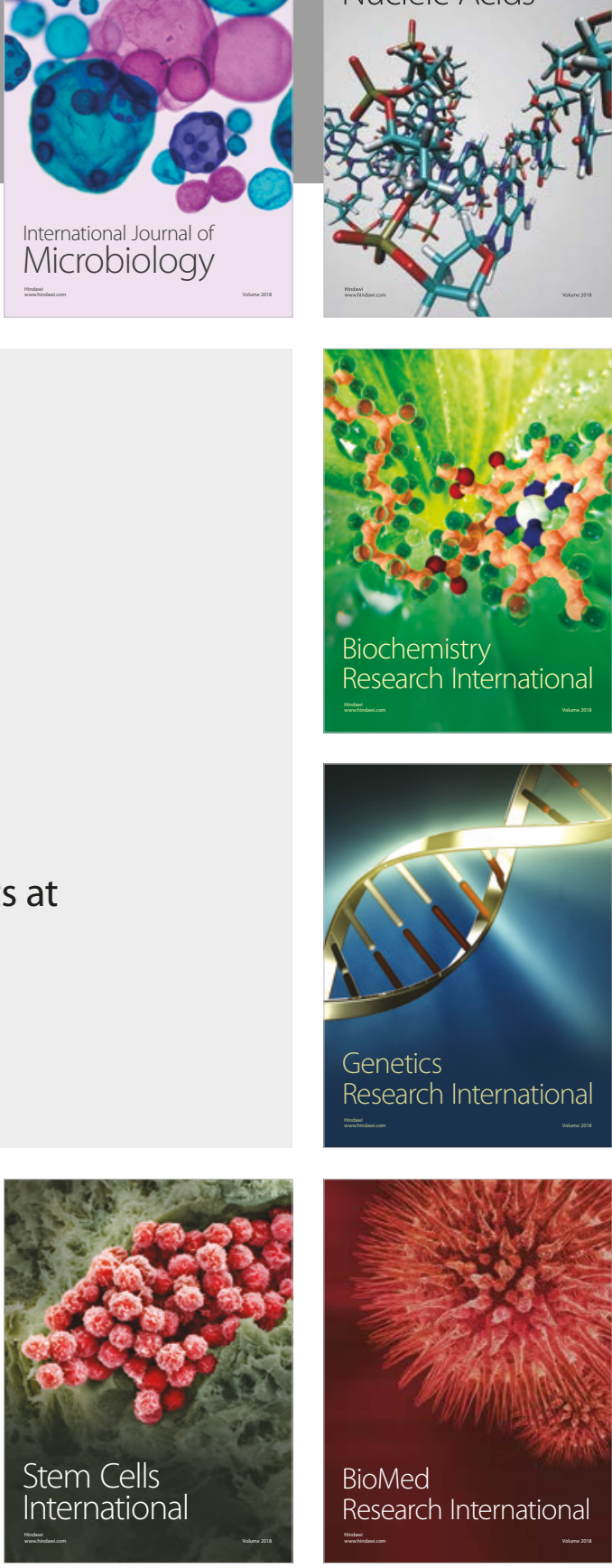
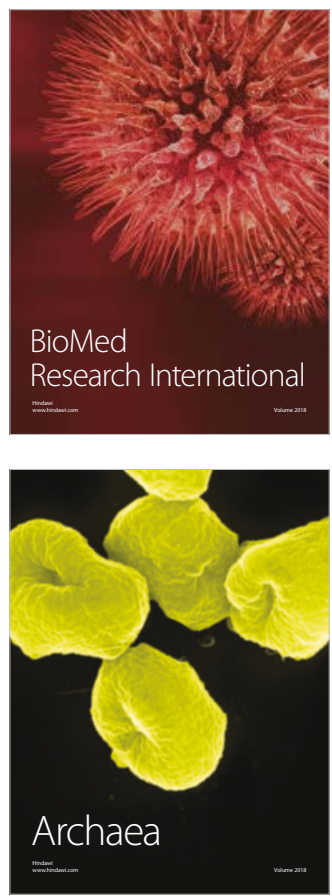